\title{
Moments of Conditional Sojourn Times in Finite Capacity M/M/1/N-PS Processor Sharing Queues
}

\author{
Nail Akar, Member, IEEE
}

\begin{abstract}
Moments of sojourn times conditioned on the length of an admitted job are derived for a finite capacity $M / M / 1 / N$ PS processor sharing queue. The mean conditional sojourn time is given in closed form whereas an expression is provided for the conditional variance in such systems involving matrix-vector operations.
\end{abstract}

Index Terms-Processor sharing queue, conditional sojourn time.

\section{INTRODUCTION}

$\mathbf{P}$ ROCESSOR sharing (PS) queues have successfully been used to model computer and communication systems in which jobs are served simultaneously by a single processor with a variable service rate depending on the total number of jobs in the system. In the most basic processor sharing discipline, called egalitarian processor sharing (EPS), the fixed total processor capacity is distributed evenly among the jobs in the system. One widespread application of EPS queues is when jobs are associated with file transfers over a telecommunications link with fixed bandwidth. A job arrives when a file transfer is initiated and the job departs when the file transmission has completed. The link bandwidth is assumed to be equally shared by all active file transfers, a property which is present when the Transmission Control Protocol (TCP) is used by hosts that initiate file transfers and when all round trip times are identical. As an example, an M/G/1-PS model has been proposed in [1] to model bandwidth sharing under TCP congestion control. Admission control has been proposed as a means to control TCP level throughput especially in overload situations leading to $\mathrm{M} / \mathrm{G} / 1 / \mathrm{N}-\mathrm{PS}$ queuing models [1],[2]. Unconditional sojourn time distributions and/or moments as well as sojourn times conditioned on the service requirement are crucial for the performance assessment of processor sharing queues with or without admission control. Besides sojourn times, average job service rates have also been explored in the literature [3]. Our focus in this letter is on the finite capacity $\mathrm{M} / \mathrm{M} / 1 / \mathrm{N}-\mathrm{PS}$ model in which the job sizes are exponentially distributed and admission control is employed that allows at most $N$ jobs in the system.

For the infinite capacity M/M/1-PS queue, closed form expressions are derived for the Laplace-Stieltjes Transform (LST) of the sojourn time distribution conditioned on the service requirement and also on the number of jobs seen

Manuscript received November 11, 2011. The associate editor coordinating the review of this letter and approving it for publication was I.-R. Chen.

This work is supported in part by the Science and Research Council of Turkey (Tübitak) under project grant EEEAG-111E106

N. Akar is with the Electrical and Electronics Engineering Department, Bilkent University, Bilkent 06800, Ankara, Turkey (e-mail: akar@ee.bilkent.edu.tr).

Digital Object Identifier 10.1109/LCOMM.2012.020212.112310 upon arrival [4]. For the M/M/1/N-PS queue, the unconditional sojourn time distribution is obtained algorithmically using Laplace transform inversion in [5]. In this letter, we address the problem of finding the sojourn time moments in an $\mathrm{M} / \mathrm{M} / 1 / \mathrm{N}$ PS queue conditioned on the length of an admitted job. We provide the mean conditional sojourn time in closed form whereas an expression based on matrix-vector operations is proposed for the conditional variance in $M / M / 1 / N-P S$ systems. Higher order moments can be obtained using Laplace transform inversion, which is left outside the scope of this letter.

The derivation of conditional sojourn times is based on the connection between processor sharing queues and Markov fluid queues [6]. A Markov fluid queue comprises an infinite size buffer that is occupied by a fluid. Let $X(t)$ denote the amount of the fluid in the buffer at time $t$. A background process, $Z(t)$, which is a finite state-space continuous-time Markov chain, modulates the buffer through a drift function $r(Z(t))$. For this fluid queue, we have

$$
\frac{d}{d t} X(t)= \begin{cases}r(Z(t)) & \text { if } X(t)>0 \\ \max \{r(Z(t)), 0\} & \text { if } X(t)=0 .\end{cases}
$$

Let $Z(t)$ have the state space $S=\{1, \ldots, M\}$ and $P$ denote its infinitesimal generator. We also define the diagonal matrix of drifts $R=\operatorname{diag}\{r(1), \ldots, r(M)\}$. Such a fluid queue is said to be characterized with the matrix pair $(P, R)$. The current letter focuses on the transient behavior of the fluid queue in the special case when $r(i)>0$ for all $i \in S$. Assume a vehicle that must traverse a link of length $x$ whose speed is governed by the background process $Z(t)$ on the finite state space $S$. Let $q_{i}$ denote the probability of the vehicle finding the background process in state $i$ just when the motion is about to start. Also let $q=\left[q_{1}, q_{2}, \ldots, q_{M}\right]$. We are interested in finding the moments of $T(x)$ which is defined as the time to travel for the vehicle that traverses a link of length $x$. At $t=0, X(t)=0$ and the motion starts. Motion ends immediately when $X(t)$ hits $x$, i.e., when $X(T(x))=x$. Let $\mu_{i}(x)=E\left[T(x)^{i}\right], i \geq 1$ and $\tilde{\mu}_{i}(s)=\int_{0}^{\infty} e^{-s x} d \mu_{i}(x)$. It was shown in [7] that

$$
\tilde{\mu}_{i}(s)=i ! q(s R-P)^{-i} e, i \geq 1,
$$

where $e$ denotes a column vector of ones of appropriate size. One of the goals of this letter is to use this relationship obtained for transportation systems in deriving the conditional sojourn times in $\mathrm{M} / \mathrm{M} / 1 / \mathrm{N}-\mathrm{PS}$ processor sharing systems.

\section{The M/M/1/N-PS Processor Sharing QueUE}

In the $\mathrm{M} / \mathrm{M} / 1 / \mathrm{N}-\mathrm{PS}$ system, jobs arrive according to a Poisson process with rate $\lambda$. EPS is used with a total service rate of unity. The system allows at most $N$ waiting jobs. Therefore, new job arrivals are blocked when there are $N$ 
jobs in the system. The service time requirement for a job is exponentially distributed with parameter $\mu$ when the server (with unity capacity) would be dedicated to this particular job only. Let $\rho=\lambda / \mu$. Two performance measures of interest are relevant to this letter: $P_{B}$ denotes the job blocking probability whereas $W(x)$ denotes the sojourn time of an arbitrary admitted job with a service requirement of $x$. The conditional moments of sojourn time are defined by $m_{i}(x)=E\left[W(x)^{i}\right]$. The conditional sojourn time variance is denoted by $\sigma^{2}(x)=$ $m_{2}(x)-m_{1}(x)^{2}$. The $i$ th unconditional moment of the sojourn time is denoted by $m_{i}=\int_{0}^{\infty} m_{i}(x) \mu e^{-\mu x} d x, i \geq 1$.

Let $Y(t)$ denote the number of jobs in the system at time $t$ in the M/M/1/N-PS queue. Let $p_{n}=\lim _{t \rightarrow \infty} P(Y(t)=$ $n), 0 \leq n \leq N$. It is well-known that $p_{n}=\rho^{n} / \sum_{i=0}^{N} \rho^{i}$ [8]. Therefore, we simply write $P_{B}=\rho^{N} / \sum_{i=0}^{N} \rho^{i}$. Consider a tagged job with a service time requirement of $x$ given that this job not blocked. This job will then find $l$ jobs upon admission (including itself) with probability $\pi_{l}=\rho^{l-1} / \sum_{i=1}^{N} \rho^{i-1}$, $1 \leq l \leq N$. Let $\pi=\left[\pi_{1}, \pi_{2}, \ldots, \pi_{N}\right]$. Once the tagged job starts service, the service rate dedicated to the tagged job will be modulated based on the background process $Y(t)$ that is governed by a continuous-time Markov chain with infinitesimal generator $Q$ of the form

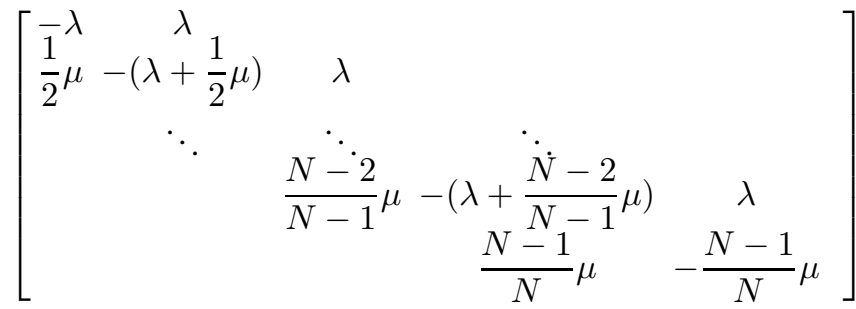

Moreover, the rate of the tagged job is given by $v(i)=\frac{1}{i}$ when there are overall $i, 1 \leq i \leq N$ jobs in the system. We define $V=\operatorname{diag}\{v(1), \ldots, v(N)\}$. The conditional sojourn time of the tagged job with service requirement of $x$ for this system is analogous to the link travel time of the vehicle that must traverse a length of $x$. Defining the LST transform $\tilde{m}_{i}(s)=$ $\int_{0}^{\infty} e^{-s x} d m_{i}(x), i \geq 1$ and using the analogy above, we have

$$
\tilde{m}_{i}(s)=i ! \pi(s V-Q)^{-i} e, i \geq 1 \text {. }
$$

The expression does not lend itself to a closed form expression for $m_{i}(x)$ for general $i$ due to the need for inverse Laplace transforms. For this purpose, we start with $m_{1}(x)$. Let $A=$ $Q V^{-1}$ and $I$ denote an identity matrix of suitable size. In this case,

$$
\begin{aligned}
\tilde{m}_{1}(s) & =\pi(s V-Q)^{-1} e=\pi V^{-1}(s I-A)^{-1} e, \\
& =\pi V^{-1}\left(\frac{I}{s}+\frac{A}{s^{2}}+\frac{A^{2}}{s^{3}}+\ldots\right) e=\frac{\pi V^{-1} e}{s}
\end{aligned}
$$

The last identity follows from $\pi V^{-1}$ being a left null vector of $Q$, which is not difficult to show. Using this identity, for $x \geq 0$, we have

$$
\begin{aligned}
m_{1}(x) & =\int_{0}^{x} \pi V^{-1} e d t=\frac{\sum_{l=0}^{N-1}(l+1) \rho^{l}}{\sum_{l=0}^{N-1} \rho^{l}} x, \\
& =\underbrace{\frac{1}{1-\rho}-\frac{N \rho^{N}}{1-\rho^{N}}}_{m})
\end{aligned}
$$

We observe that the conditional mean sojourn time in the finite capacity case is linear in $x$ as in its infinite capacity counterpart for which $m_{1}(x)=\frac{1}{1-\rho} x$ [4]. The factor in this linear form is the mean number of jobs $m$ upon arrivals of admitted jobs. Note that in this definition, the newly admitted job is also taken into account. Moreover, there is a reduction in the mean sojourn time given by the term $\frac{N \rho^{N}}{1-\rho^{N}} x$ stemming from rejection of jobs in overload situations compared to infinite capacity case. This closed form expression can be used to dimension finite capacity processor sharing systems.

For the second moment, we have $\tilde{m}_{2}(s)=$

$$
2 \pi(s V-Q)^{-2} e=\frac{2 \pi V^{-2}(s I-A)^{-1} e}{s},
$$

which leads us to

$$
m_{2}(x)=\int_{t_{1}=0}^{x} \int_{t_{2}=0}^{t_{1}} 2 \pi V^{-2} e^{A t_{2}} d t_{2} d t_{1} .
$$

It is preferable to avoid numerical integration. Therefore, we propose the following method to obtain the second moment of the conditional sojourn time. For this purpose, note that the matrix $Q$ is a stochastic matrix and has a single eigenvalue at zero. Therefore, $A$ has a single eigenvalue at zero as well. The left and right null vectors of $A$ are denoted by $v$ and $u$, respectively. In particular, $v=\frac{1}{\sum_{i=1}^{N} \rho^{i-1}}\left[1,2 \rho, \ldots, N \rho^{N-1}\right]$ and $u=[1,1 / 2, \ldots, 1 / N]^{T}$ satisfying $v u=1$. Also let $B=A-u v$, which is obtained my moving the eigenvalue of $A$ at zero to minus one. Thus, $B$ is an invertible matrix. With these definitions, it is not difficult to show that $A^{n}=B^{n}+(-1)^{n} u v, n \in \mathbb{Z}^{+}$and moreover $e^{A t}=$ $e^{B t}+u v\left(1-e^{-t}\right), t \in \mathbb{R}^{+}$. Noting that $\frac{d}{d t} B^{-1} e^{B t}=e^{B t}$ and applying straightforward rules of integration for the equation (4), we finally obtain

$$
\begin{aligned}
\sigma^{2}(x) & =2 m^{2}\left(1-x-e^{-x}\right) \\
& +2 \pi V^{-2}\left(B^{-2} e^{B x}-B^{-2}-B^{-1} x\right) e .
\end{aligned}
$$

The identity above allows one to write down the conditional variance of the sojourn time using matrix-vector operations. Also note that the unconditional moments are given by

$$
m_{i}=\tilde{m}_{i}(\mu)=i ! \pi(\mu V-Q)^{-i} e .
$$

In particular, $m_{1}=m / \mu$.

\section{EXTENSION TO Generalized Processor SHARING}

The overall service capacity being $f_{i}$ when there are $i$ jobs in the system is referred to as generalized processor sharing (GPS) [5]. Note that the special case $f_{i}=1, \forall i$ reduces to EPS. It has been shown in [9] that the userlevel performance of the popular proportional fair scheduling algorithm in opportunistic cellular wireless networks may be evaluated using a GPS model in which the total service rate is not fixed but instead increases with the total number of users. This stems from the observation that it would be more likely to select a user with high transmission rate out a larger user population. It is not difficult to show that the previous analysis is readily extensible to GPS. For this purpose, consider an 


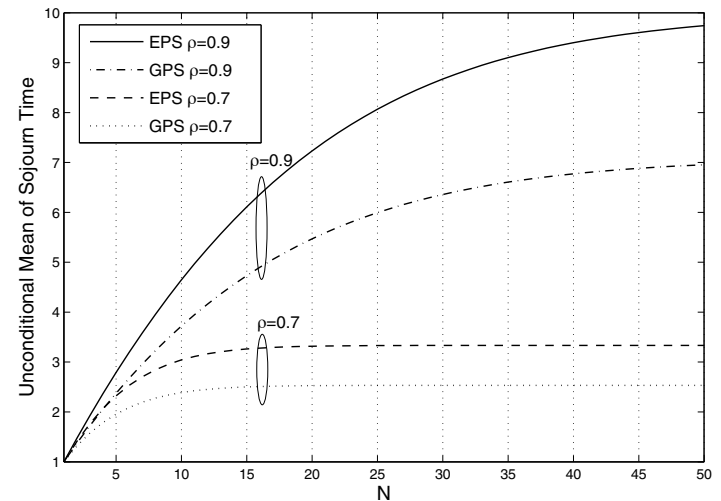

Fig. 1. Unconditional mean sojourn times as a function of $N$ in EPS and GPS systems for two different values of $\rho=0.7,0.9$.

admitted job which will see $l, 1 \leq i \leq N$ jobs (including itself) upon admission with probability [5]:

$$
\pi_{l}^{(G)}=\frac{\rho^{l-1} \phi_{l-1}}{\sum_{l=1}^{N} \rho^{l-1} \phi_{l-1}}, \phi_{i}=\frac{1}{\prod_{k=1}^{i} f_{k}}, i>0, \phi_{0}=1 .
$$

Let $\pi^{(G)}=\left[\pi_{1}^{(G)}, \pi_{2}^{(G)}, \ldots, \pi_{N}^{(G)}\right]$. Once the tagged job starts service, the service rate the tagged job receives depends on the state of a Markov chain with infinitesimal generator $Q^{(G)}$ which can be obtained through $Q$ by replacing the $\mu$ term in the $j$ th row of $Q$ by $f_{j} \mu$. The rate of the tagged job is given by $v^{(G)}(i)=\frac{f_{i}}{i}$ when there are overall $1 \leq i \leq N$ jobs in the system. We define $V^{(G)}=\operatorname{diag}\left\{v^{(G)}(1), \ldots, v^{(G)}(N)\right\}$. We define $\tilde{m}_{i}^{(G)}(s)=\int_{0}^{\infty} e^{-s x} d m_{i}^{(G)}(x), i \geq 1$ where $m_{i}^{(G)}(x)$ is the $i$ th moment of the conditional sojourn time of the tagged job with service requirement $x$ under GPS. Using again the results of [7], one obtains

$$
\tilde{m}_{i}^{(G)}(s)=i ! \pi^{(G)}\left(s V^{(G)}-Q^{(G)}\right)^{-i} e, i \geq 1 .
$$

In particular, $m_{1}^{(G)}(x)=m^{(G)} x$ where

$$
m^{(G)}=\frac{\sum_{l=1}^{N} l \rho^{l-1} \phi_{l-1}}{\sum_{l=1}^{N} \rho^{l-1} \phi_{l-1}}
$$

denotes the expected number of jobs that an arriving job sees just after the job is accepted into the GPS system. On the other hand, the conditional variance of the GPS system can be obtained using the method described for EPS.

\section{NUMERICAL EXAMPLES}

In the first example, we present results concerning an $\mathrm{M} / \mathrm{M} / 1 / \mathrm{N}-\mathrm{PS}$ queue under EPS and GPS. For GPS, we use $f_{i}=\sum_{j=1}^{i} 1 / j$ as suggested in [5] which is motivated by a wireless channel-aware downlink scheduler under Rayleigh fading [9]. We fix $\mu=1$ and the unconditional expected sojourn times are plotted as a function of $N$ in Fig. 1 for two different values of $\rho=0.7,0.9$. The unconditional expected sojourn time decreases with reduced $N$ and also with GPS in relation with EPS due to the opportunistic nature of the scheduler.

In the second example, the conditional variance $\sigma^{2}(x)$ is plotted in Fig. 2 against the job length for various values

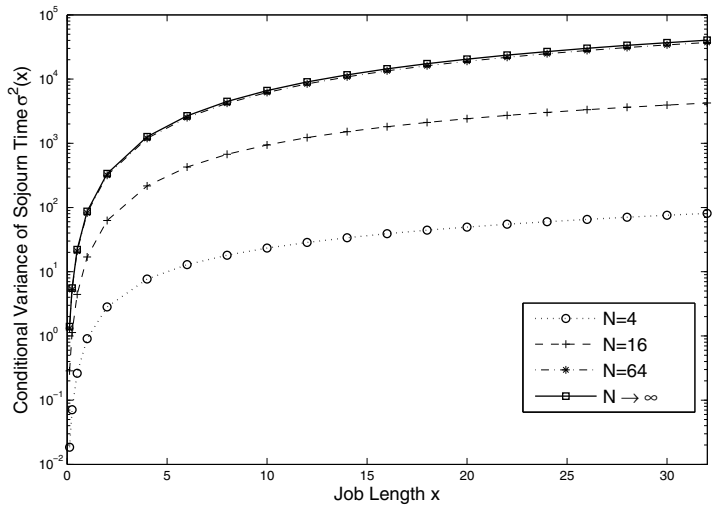

Fig. 2. Conditional variance of the sojourn time as a function of job length $x$ for an M/M/1/N-PS system for $\rho=0.9$ for various values of $N$.

of $N$ for the case $\rho=0.9$ in the case of EPS. The conditional variance for the case $N \rightarrow \infty$ is obtained using the expression provided in [4] whereas results for finite values of $N$ are obtained using the expression (5). The finite capacity results almost overlap with those of the M/M/1/-PS system for $N=64$. Therefore, we are inclined to believe that the finite capacity processor sharing queue conditional moments can also be used to approximate those of its infinite capacity counterpart with suitably large choices of $N$.

\section{CONCLusion}

Unlike most existing work on infinite capacity systems, we provide expressions for the moments of sojourn times conditioned on the length of admitted jobs for finite capacity $\mathrm{M} / \mathrm{M} / 1 / \mathrm{N}-\mathrm{PS}$ processor sharing queues with the expression being in closed form for the conditional mean. Such expressions can be used in provisioning servers employing admission control with EPS or GPS capacity sharing.

\section{REFERENCES}

[1] L. Massoulie and J. Roberts, "Bandwidth sharing and admission control for elastic traffic," Telecommunication Systems, vol. 15, no. 1-2, pp. 185201, 2000.

[2] S. Shenker, "Fundamental design issues for the future Internet," Selected Areas in Communications, IEEE Journal on, vol. 13, no. 7, pp. 1176 1188, sept. 1995.

[3] N. Chen and S. Jordan, "Violation probability in processor sharing queues," IEEE Trans. Auto. Cont., vol. 53, no. 8, pp. 1956-1961, 2008.

[4] E. G. Coffman, Jr., R. R. Muntz, and H. Trotter, "Waiting time distributions for processor-sharing systems," J. ACM, vol. 17, pp. 123-130, January 1970.

[5] S. Borst, O. Boxma, and N. Hegde, "Sojourn times in finite-capacity processor-sharing queues," in Next Generation Internet Networks, 2005, 2005, pp. $53-60$.

[6] D. Anick, D. Mitra, and M. M. Sondhi, "Stochastic theory of a data handling system with multiple sources," Bell Syst. Tech. Jour., vol. 61, pp. 1871-1894, 1982.

[7] J. P. Kharoufeh and N. Gautam, "A fluid queueing model for link travel time moments," Naval Research Logistics (NRL), vol. 51, no. 2, pp. 242257, 2004.

[8] J. W. Cohen, "The multiple phase service network with generalized processor sharing," Acta Informatica, vol. 12, pp. 245-284, 1979.

[9] S. Borst, "User-level performance of channel-aware scheduling algorithms in wireless data networks," IEEE/ACM Trans. Netw., vol. 13, pp. 636-647, June 2005. 